\title{
Estudio 50 Casos de Colpotomía Anterior para Ligadura Tubaria
}

\author{
Jorge E. Zúñiga *
}

\section{RESUMEN}

Se presenta un estudio de 50 pacientes con ligadura de trompas transvaginal por el método de la colpotomía anterior, usando la técnica de Madneler.

Este sistema quirúrgico ha sido muy utilizado, como método definitivo para hacer planificación familiar, especialmente en la India.

En los 50 casos, realizados en el servicio de ginecología del Hospital Universitario de Cali,Colombia S.A. se hace notar la gran aceptabilidad del método por las pacientes; la técnica operatoria es senciIla, rápida, con instrumental común y corriente; la recuperación rápida en el post-operatorio, la estancia mínima hospitalaria; la seguridad del $99 \%$ de los casos, con la única condición de cirujano hábil y con experiencia en cirugíavaginal.

La técnica para esterilización femenina por ligadura de trompas se remonta a los principios del siglo XIX.

Consiste en una incisión a través de la vagina, en lugar del abdómen, la cual

\footnotetext{
Profesor asociado

Jefe Servicio de Ginecologia

Depto. Obstetricia y Ginecología

División de Salud

Universidad del Valle

Cali - Colombia - S.A.
}

resulta especialmente eficaz en la esterilización tubaria.

Recientemente, este método ha sido utilizado especialmente en la India (1,2, $3,4)$.

El procedimiento es bastante sencillo; la paciente recibe anestesia regional (Epidural-Peridural ó Raquídea) y neurolepto-analgesia (entre nosotros). La mayoría de las pacientes pueden salir del hospital el mismo día o el día siguiente de la intervención.

La morbilidad y las complicaciones mayores son mínimas.

Ultimamente un grupo de expertos reunidos en Bombay en 1973, se pronunció en favor de la colpotomía, afirmando que produce menos dolor post-operatorio $y$ "no despierta el mismo temor que una intervención que exija una incisión abdominal" (5).

\section{Ventajas de la colpotomía}

1. Puede llevarse a cabo sin estancia hospitalaria, o ésta puede ser muy corta.

2. El tiempo operatorio promedio es de 15 minutos.

3. Basta utilizar anestesia regional.

4. Hay menos dolor post-operatorio.

5. No hay incisión abdominal y por lo tanto no queda cicatriz visible. 
6. Los instrumentos necesarios son sencillos y se encuentran en cualquier servicio de cirugía.

7. No hay necesidad de electrocoagulación.

8. Las tasas de morbilidad son mínimas.

9. La tasa de fracaso es solo del $1 \%$.

10.Se puede combinar con otros procedimientos ginecológicos (Corrección del cistocele y de la incontenencia urinaria).

11. La intervención es fácil y segura.

\section{Resumen Histórico}

Se cree que fue J. Alfred Recamier quien hizo la primera colpotomía en 1831, por absceso pélvico (6). En 1895 A. Duhrsen describió la técnica (7). W.W. Babcoock en 1928 la recomendó para ciertas operaciones ginecológicas, para obviar el riesgo de la laparotomía (8). En 1943 B.N. Purandare sugirió la colpotomía como método de esterilización a través del fondo de saco vaginal anterior (9). Desde entonces hasta el momento se han informado muchas series de pacientes con este procedimiento, en varias áreas del mundo. $(10,11,12,13,14$, 15).

\section{Preparación Preoperatoria}

Es diferente en los distintos sitios del mundo; en la mayoría de los casos las pacientes ingresan al Hospital el día anterior a la intervención. En general debe seguirse el siguiente proceso de estudio y manejo:

Historia Clínica

Exámenes de laboratorio rutinarios

Examen físico

Asepsia del periné $y$ vagina con soluciones o supositorios bactericidas 0 bacteriostáticos.

Muchos cirujanos no están de acuerdo con utilizar supositorios vaginales ni du- chas bactericidas, porque consideran imposible esterilizar la vagina. Sin embargo, algunos utilizan duchas vaginales con soluciones anti-sépticas la víspera de la intervención (16) y otros prefieren hacer las medidas de asepsia en el momento mismo de la cirugía (17).

En nuestras pacientes el procedimiento de preparación que se siguió, además de las medidas ya expuestas, fue utilizar un supositorio vaginal diario con Cloranfenicol durante los 6 días previos al procedimiento.

Podríamos afirmar, con base en nuestra experiencia, que los pasos mínimos de preparación son los mencionados anteriormente.

\section{Anestesia}

Se puede emplear anestesia local, raquídea o general. Cada una tiene ventajas y desventajas específicas. Creemos es importante la relajación. En nuestras pacientes siempre se utilizó anestesia epidural.

Parece ser la anestesia raquídea la mas frecuentemente empleada en la India. En los Estados Unidos se utiliza con más frecuencia la anestesia general. "En los países desarrollados se emplean dosis mayores de anestesia que en los países en vía de desarrollo".

\section{Técnica quirúrgica de la colpotomía anterior.}

Se trata de alcanzar las trompas a través del fondo de saco vesico-uterino, en lugar de hacerlo por el Douglas; asi se hace frecuentemente en el Japón.

La intervención se inicia con una incisión vertical de aproximadamente $3 \mathrm{cms}$ de extensión, en el fondo de saco anterior y sobre la mucosa vaginal (Fig. 1). Se hace la disección del tejido conjuntivo situado entre la vejiga y el cuello uterino. Se desplaza la vejiga y se toma el peritoneo entre pinzas (Fig. 2) y se abre. 
Figuras 1 y 2

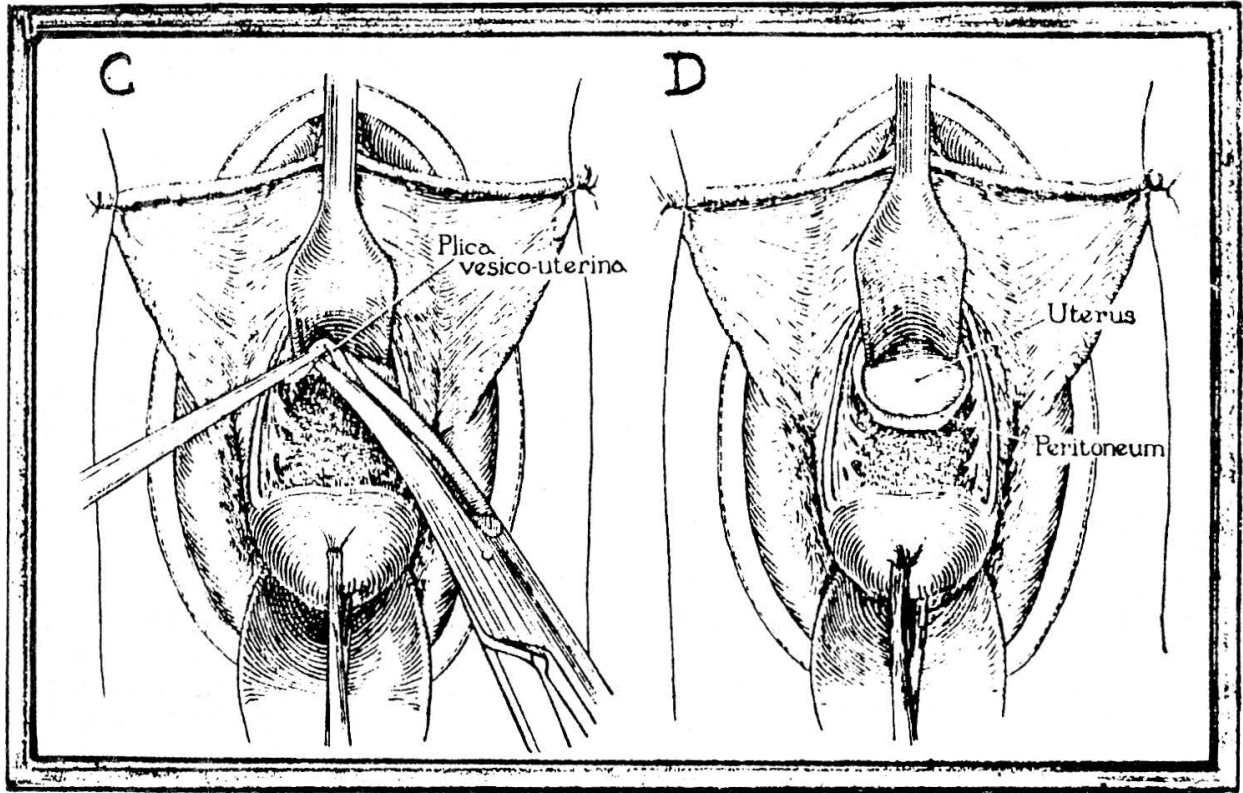

Dibujos tomados de R.W. Telinde; Operative Ginecology, Filadelfia.

Al aparecer el útero es fácil tomar las trompas con pinzas de corazón y de Babcok; se hace un asa cerca de la fimbria y se ligan seccionándolas por el método de Madlener en nuestro estudio, colocando doble ligadura con seda (Figura 4).

La operación termina con la sutura del peritoneo y de la mucosa vaginal con Cat-Gut Crómico 00 ó 0 . Si hay Cistocele se aprovecha para corregirle.

"La cirugía debe ser rápida, suave y realizada con destreza".

\section{Cuidados post-operatorios}

En general son mínimos:

La paciente permanece en reposo de 4 a 6 horas. Se controlan signos vitales. Se ordena dieta líquida para el primer día, analgésicos y supositorios con antibióticos para 1 semana.

El coito debe evitarse durante 4-6 semanas.

El período de hospitalización es de 1 día y la operación puede hacerse en programas de Medicina Ambulatoria, con cuidados post-operatorios en casa (18, $19,20,21,22,23)$.

Otros médicos permiten mas estancia hospitalaria, más si se practican conjuntamente otras intervenciones ginecológicas.

La tendencia hoy día va hacia una permanencia hospitalaria muy corta (24, 25).

\section{Instrumental}

El instrumental necesario es de fácil obtención y consta de: 
Una valva de peso

Una pinza de cuello

Dos pinzas de Allix

Dos retractores o separadores vaginales.

Una pinza Babcock

Una pinza de corazón curva

Instrumental de corte (bisturi, tijeras, Metzenbaum).

Material de sutura.

$\mathrm{Si}$ es factible se recomienda un retrovector uterino para movilizar el útero; nosotros utilizamos una cánula de hidrotubación en algunos casos, ya que con las pinzas de corazón se localizan con mucha facilidad las trompas.

Creemos este instrumental se encuentra en cualquier servicio de cirugía.

\section{Contraindicaciones:}

1. Utero fijo (adherencias)

2. Infección pélvica

3. Masas o tumores anexiales

4. Obesidad extrema

5. Vagina estrecha y profunda o vagina estenótica.

\section{Condiciones ideales para el procedi- miento}

1. Utero de tamaño normal, completamente móvil $y$ en posición conveniente.

2. Anexos sanos y no adheridos

3. Fondos de saco libres

4. Vagina relajada de multípara

5. Conducto genital en buen estado

6. Cirujano capacitado (26)

\section{Morbilidad y complicaciones}

En la India las tasas de morbilidad son de 1,6 y $5.55 \%$; en Europa y en Estados Unidos van desde el $3,3 \%$ al 13 , $3 \%$
Las causas más comunes de morbilidad son la infección y la hemorragia. La obesidad es un factor agravante, (27) así como también las relaciones sexuales precoces.

La infección se presenta por técnica imperfecta $v / o$ falta de asepsia durante el acto operatorio. Se pueden presentar abscesos y aún pelviperitonitis o peritonitis, que implican cuidado intensivo del paciente y laparotomía.

En el grupo de pacientes que presentamos en este estudio, lo único que se presentó en algunos casos fue leve dolor pélvico en el post-operatorio inmediato.

\section{MATERIAL Y METODOS}

Hemos realizado el procedimiento en 50 pacientes, quienes lo solicitaron voluntariamente y previa aceptación del cónyuge, provenientes de la Clínica de Planificación Familiar del Hospital Universitario del Valle.

Una vez confeccionada la Historia Clínica, las pacientes tenían entrevista con la Trabajadora Social del Programa. El manejo pre-operatorio se hizo a base de supositorios vaginales con Cloranfenicol por 6 días. El procedimiento se realizó con anestesia epidural en todos los casos. La hospitalización en todos los casos tuvo una duración de 24 horas; creemos puede ser mucho mas corta. El tiempo promedio de cirugía fluctuó entre 15 y 25 minutos.

Como técnica quirúrgica se usó la colpotomía anterior, ya descrita, utilizando para la ligadura de las trompas la técnica de Madlener, colocando doble ligadura con seda.

En el post-operatorio se continuaron los óvulos vaginales con cloranfenicol por 6 días más; se prohibieron relaciones sexuales durante 4 a 6 semanas. En los controles, las pacientes han evolucionado en forma perfecta y la morbilidad y complicaciones no se presentaron en ninguno de los casos. 
A todas las pacientes se les hizo estudio histológico de la sección de las trompas extirpadas, informándose siempre como normal el tejido estudiado; hasta el momento de escribir estas notas no hemos tenido el primer fracaso y ya los primeros casos tienen aproximadamente 1 año.

Es de anotar que en el post-operatorio la pareja y especialmente la mujer, se ha sentido muy satisfecha con el método; creemos que el hecho de no ver herida abdominal influye en la recuperación rápida de todos los casos; muchas pacientes solicitaron salir del hospital el mismo día de la intervención.

En el cuadro No. 1 se presenta el total de pacientes por grupos de edad; es de anotar que el mayor porcentaje de casos está en el grupo de 31 a 35 años, para $48 \%$ del total, como que a esta edad ya se tienen el número de hijos deseado en cada caso.

Cuadro No. 1

\section{GRUPOS DE PACIENTES POR EDAD}

\begin{tabular}{|c|c|c|c|c|c|}
\hline $\begin{array}{c}\text { Edad } \\
\text { en } \\
\text { Años }\end{array}$ & $20-24$ & $25-30$ & $31-35$ & $36-40$ & 40 \\
\hline $\begin{array}{c}\text { No. de } \\
\text { pacientes }\end{array}$ & 2 & 11 & 24 & 11 & 2 \\
\hline$\%$ & 4 & 22 & 48 & 22 & 4 \\
\hline
\end{tabular}

En el cuadro No. 2 se muestran las pacientes de acuerdo a la gravidez. Corresponde el mayor porcentaje de casos para el grupo 7-10 embarazos, el $50 \%$.

Cuadro No. 2

\section{GRUPO DE PACIENTES POR GRAVIDEZ}

\begin{tabular}{|c|c|c|c|c|}
\hline $\begin{array}{c}\text { No.de } \\
\text { embarazos }\end{array}$ & $1-3$ & $4-6$ & $7-10$ & 10 \\
\hline $\begin{array}{c}\text { No. de } \\
\text { pacientes }\end{array}$ & 2 & 15 & 25 & 8 \\
\hline$\%$ & $4 \%$ & $30 \%$ & $50 \%$ & $16 \%$ \\
\hline
\end{tabular}

En el cuadro No. 3 se agrupan las pacientes por paridad. Corresponde el mayor número de casos al grupo entre los 4 y 6 partos, para un $52 \%$.

\section{Cuadro No. 3}

\section{GRUPO DE PACIENTES SEGUN PARIDAD}

\begin{tabular}{|c|c|c|c|c|}
\hline $\begin{array}{c}\text { No. de } \\
\text { partos }\end{array}$ & $1-3$ & $4-6$ & $7-10$ & 10 \\
\hline $\begin{array}{c}\text { No. de } \\
\text { pacientes }\end{array}$ & 3 & 26 & 20 & 1 \\
\hline$\%$ o & 6 & 52 & 40 & 2 \\
\hline
\end{tabular}

En el cuadro No. 4 se presentan las causas por las cuales las pacientes decidieron la ligadura de trompas.

Cuadro No. 4

\begin{tabular}{|c|c|}
\hline Intolerancia a gestágenos orales ....... 7 & \\
\hline Intolerancia a $D \mid \cup \ldots \ldots \ldots \ldots \ldots 6$ & \\
\hline Intolerancia a gestágenos orales y $D \perp \cup \ldots 10$ & \\
\hline azos con $D \mid \cup \ldots \ldots \ldots \ldots 3$ & \\
\hline ridad como única indicación . . 20 & \\
\hline a columna cervical . . . . . . 1 & \\
\hline tosis . ........... 1 & \\
\hline$\ldots \ldots \ldots 1$ & \\
\hline Por hipertensión arterial crónica ....... 1 & \\
\hline Por esquizofrenia $\ldots \ldots \ldots \ldots \ldots \ldots 1$ & \\
\hline Porobacidad & $7.3 \%$ \\
\hline
\end{tabular}

Se hace notar que la gran mayoría de las pacientes habian tenido alguna experiencia con otros métodos para planificar la familia $y$ en algunos casos habían tenido fracasos de los mismos ó fenómenos de intolerancia severos.

\section{CONCLUSIONES}

1. Se presenta el estudio de 50 pacientes con ligadura de trompas trans-vaginal por el método de la colpotomía anterior usando la técnica de Madlener.

2. Como preparación pre-operatoria se usaron supositorios vaginales por 6 días a base de Cloranfenicol. 
3. Se utilizó anestesia epidural.

4. La estancia hospitalaria fue de 24 horas.

5. La morbilidad y las complicaciones no se presentaron en este grupo.

6. El mayor porcentaje de casos correspondió a edades entre 31 y 35 años $(48 \%)$.

7. El mayor número de casos tenía de 4 a 6 partos, para un $(52 \%)$.

8. Las principales indicaciones fueron la multiparidad y la intolerancia a otros métodos.
9. Se anota la gran aceptabilidad del método por las pacientes.

10.Se hace hincapié en la recuperación rápida $y$ el reintegro a la vida normal en tiempo relativamente corto.

11. Hasta el momento no hay fracasos del método empleado.

12. Como anotación importante creemos que el cirujano que practique el procedimiento debe tener experiencia en cirugía vaginal.

\section{CASES OF ANTERIOR COLPOTOMY FOR TUBAL LIGATION}

\section{SUMMARY}

This article summarizes the findings of a study on 50 patients with transvaginal tubal ligation by means of anterior colpotomy using the Madneler technique.

This surgical technique has been widely used as non-reversible method of contraception, especially in India.

\section{REFERENCIAS}

1. PURANDARE, V.N. Evaluation of operative method for female sterilization, in.

PURANDARE, B.N. and THAYERI, C.L., eds Actas del Seminario Internacional sobre Mortalidad Materna, Planificación Familiar y Biología de la Reproducción.

Bombay India marzo 3-8 1969-1970 P: 265269.

2. PURANDARE, B.N. Development and implementation of a Sterilization Programm. Informe presentado ante la segunda conferencia Internacional sobre Esterilización vo-
This study, conducted at the Gyn Deptartment of Cali University Hospital, shows the large acceptance of this technique by the patients. The method is simple, effective, quick and uses simple instruments. Recovery after surgery is short and women can leave the hospital soon. It is safe in $99 \%$ of the cases, as long as the operator is an experienced surgeon.

luntaria. Ginebra, Suiza, Febrero 28, 1973 P. 16.

3. SOONAWALA R. Vaginal Techniques of Surgical Sterilization, Resumen de la Primera Conferencia Médica y Científica a escala Regional de la IPPF para el Sureste Asiático y Oceanía, Sydney, Australia 14-18 1972.

Asociación de Planificación Familiar de Australia 1972 P. 49.

4. SOONAWALA, R.P. Vaginal Sterilizatión. Informe presentado ante la segunda conferencia Internacional sobre Esterilización Voluntaria, Suiza, Febrero 281973 P: 17. 
5. ANKLESARIA S.B. Statiscal Review of 5.900 female Sterilizations At The Civil Hospital Chinai Maternity Hospital and Lilubhai Gord Handas Hospital, Ahmedabad with a prospective follow-up of 1936 patients for one to five years in: Purandare, B.N. and Jhaveri, C.L. Actas del Seminario International sobre Mortalidad Materna, Planificación Familiar y Biología de la Reproducción, Bombay India, Marzo 3-8-1969-1970 P: 181194.

6.BABCOCK, W.W. The Vaginal approach for Certain Intraperitoneal Operations.

American Journal of Obstetrics and Gynecology 17:573 1928.

7.BEN-CHEIKH, T.D., HADDAD, $M$, and BOUDJEMAAS, A discusion of some cases of sterilization by colpotomy.

(Fr.) Tunisie Medicale 49 (2): 121-122.

8.BENDEL. Female Sterilización (Editorial) Minnesota Medicine 55. 235 Marzo 1972.

9. BOYSEN, H. and NcRAE, L.A. Tubal sterilización through the vagina.

Amesican Journal of Obstetrics and Gyneco. logy 58:488-493 Septiembre 1949.

10. LEONARDO, R.A. History of Gynecology. New York Froben Press - 1944. 303 P.

11. Ver No. 6

12. Ver No. 2

13. Ver No. 10.

14.JOHN, A.H. and DUNSTER, G.D. Sterilization by Posterior Colpotomy.

Journal of Obstetrics and Gynecology of the British Commonwealth 79: 381-382 abril 1973.

15.Ver No. 09.

16. ENGINEER, A.D. A Simple Method of Vaginal Sterilization. Journal of Okstetrics And
Gynecology of India. 22 (1). 38-40 Febrero 1972.

17. EVANS, T.N. Simplified Method for Sterilization of The Female. American Journal of Obstetrics and Gynecology 66: 393-395 Agosto 1953.

18.COLLINS, J.A. ALLEN H.H. and YUZPE A.A. Outpatient management of first trimester therapeutic abortions with and without tubal ligation.

Canadian Medical Association Journal 106: 1077-1080 Mayo 201972.

19. HASKINS, A.L. Oviductal sterilization with tantalum clips. American Journal of Obstetrics and Gynecology 114(3):370-377 Octubre 1, 1972 .

20.LAHIRI, B.C. and MITRA, K.N. Vaginal Iigation under local anesthesis a simplified technique, Journal Of Obstetrics and Gynecology of India 22(3): 330-333 Junio 1972.

21. MORRIS, J.A. Posterior Colpotomy. Springfield, Illinois, Charles C. Thomas 1969-57

22. SHUTE, W.B. Transvaginal sterilization: Use of a new uterine retroverter and method. American Journal of Obstetrics and Gynecology 115(7) : 998-1001 Abril 1973.

23. YUZPE, A.A. ALLEN, H.H. And COLLINS, J.A. Tubal sterilization: Methodology, postoperative management and followup of 2934 cases. Canadian Medical Association Journal 107 : 115-117 Julio 221972.

24. Ver 22.

25. Ver No. 1.

26. DAWN, C.S., Culdoscopic Tubal Ligation. Informe presentado ante la Segunda Conferencia Internacional sobre Esterilización Voluntaria Ginebra, Suiza, Febrero 281973 P 2.

27.Ver No. 31. 